\title{
AKnowledge and Attitudes Regarding Cervical Cancer Screening among Women in Debre Markos Town, Amhara Region, Northwest Ethiopia: Community Based Cross-Sectional Study
}

\author{
Bewket Yeserah Aynalem ${ }^{1 *}$, Kiber Temesgen Anteneh ${ }^{2}$ and Mihretu Molla Enyew ${ }^{2}$ \\ ${ }^{1}$ Department of Midwifery, Debre Markos University, Debre Markos, Ethiopia \\ ${ }^{2}$ Department of Midwifery, University of Gondar, Gondar, Ethiopia
}

\begin{abstract}
Introduction: Cervical cancer is the most common type of malignancy among all malignancies for women worldwide with 527,600 cases and 265,700 deaths every year. This research aimed to assess the knowledge of cervical cancer, attitude towards its screening, and associated factors among women at Debremarkos town, Northwest Ethiopia.

Methods: A community-based cross-sectional study was conducted among women from 30-49 years in Debremarkos town, from March 11 to May 31, 2020. Bivariable and multivariable logistic regression analyses were performed with SPSS version 25 and variables with a $p$-value of less than 0.05 were taken as significant variables.

Result: The study showed that $374(59.4 \%)$ and 385 (61.1\%) of respondents were knowledgeable and had a favorable attitude respectively. History of multiple sexual partners [AOR: $1.768(95 \% \mathrm{Cl}: 1.227,2.549)$ ], and modern family planning method use [AOR: 2.238 ( $95 \% \mathrm{Cl}: 1.410,3.554)$ ] were significantly associated with knowledge of cervical cancer screening.

Again, education level ([AOR: 2.006, 95\%Cl: 1.147, 3.508)]) marital status [AOR: 2.101 (95\% Cl: 1.219, 3.620)], and gravidity [AOR: $1.830(95 \% \mathrm{Cl}: 1.125,2.976)]$, were significantly associated with attitude towards cervical cancer screening.

Conclusion: This study showed the magnitude of knowledge and attitude towards cervical cancer screening was low even though the government recommended cervical cancer screening for eligible women.

Keywords

Cervical cancer, Screening, Knowledge, Attitude

List of Abbreviations

CC: Cervical cancer is Cancer; HPV: Human Papilloma Virus; LMICs: Low and Middle-Income Countries; MSPs: multiple sexual partners; STD: Sexually transmitted disease; WHO: World Health Organization
\end{abstract}

\section{Introduction}

Cervical cancer is Cancer (CC) that starts in the cervix and occurs when cells in the cervix proliferate abnormally that have the chance to spread out to other parts of the body which is $70 \%$ of the case is caused by Human Papilloma Virus (HPV) $[1,2]$. CC screening is the use of different tests on individuals who have no signs and symptoms of a disease to identify those who probably have risk factors or are in the early stage of the disease $[1,3]$. CC is a global public health concern and a chronic non-communicable disease [4].

A global study reported 527,600 CC cases and 265,700 deaths occurred and $90 \%$ of cases occurred in Low and Middle-Income Countries (LMICS); the highest was occurred in
Sub-Saharan Africa (SSA) where CC is the leading killer among women [5]. Usually, women with CC do not observe symp-

*Corresponding author: Dr. Bewket Yeserah Aynalem, Department of Midwifery, Debre Markos University, Debre Markos, Ethiopia

Accepted: February 11, 2021

Published online: February 13, 2021

Citation: Aynalem BY, Anteneh KT, Enyew MM (2021) Knowledge and Attitudes Regarding Cervical Cancer Screening among Women in Debre Markos Town, Amhara Region, Northwest Ethiopia: Community Based Cross-Sectional Study. J Nurs Pract $4(1): 240-248$ 
Citation: Aynalem BY, Anteneh KT, Enyew MM (2021) Knowledge and Attitudes Regarding Cervical Cancer Screening among Women in Debre Markos Town, Amhara Region, Northwest Ethiopia: Community Based Cross-Sectional Study. J Nurs Pract 4(1):240-248

toms, especially in the early stages [6]. Therefore, the presentation of malignancy in the late-stage is vaginal bleeding, invasion, metastasis, and poor prognosis [7]. In addition to presenting a significant burden in terms of morbidity and mortality, CC also increases economic risk, which imposes very high direct costs on health systems, communities, households, and lost productivity of patients, premature death, and disability. The occurrence of CC related death is rare in high-income countries because of effective pre-invasive CC screening, and the risk of dying from CC is higher in resource-poor countries [8].

Cervical carcinoma is still the leading cause of morbidity and mortality among women on the African continent [9]. In Ethiopia, 7095 cases and 4732 death of CC occurs every year [10]. It is considered preventable because it has a long pre-invasive state to be changed from pre-cancer lesion to cancer and this gives a big chance to screen pre-cancer lesion and stop the existence of CC $[2,11]$.

World Health Organization (WHO) recommended age for CC screening should be limited to women in the age of 30 to 50 years since there is evidence on younger age women with a milder degree of lesion spontaneously recover to normal [1]. As the WHO information Centre report showed there is a great difference in the level of CC coverage in different countries and the highest effective coverage was found in Ukraine (78\%), the lowest was found in Ethiopia (1\%) [12]. Having good knowledge and a positive attitude on CC screening is linked with the availability of health services $[13,14]$.

Ethiopia adopted the WHO guideline and advised women to start CC screening at age of 30-49 years [1,7,15]. But CC utilization may be affected by women's knowledge and attitude towards CC screening [16]. Thus the purpose of this research is to assess the knowledge of cervical cancer, attitude towards its screening, and associated factors among women at Debremarkos town, Northwest Ethiopia.

\section{Methods}

\section{Study area and period}

This study was done in Debremarkos town, Amhara region, Northwest Ethiopia from March 11 to May 31, 2020. Debre Markos town is the capital city of East Gojjam Zone in Amhara regional state, Northwest Ethiopia, which is located at $300 \mathrm{~km}$ from Addis Ababa, the capital city of Ethiopia, and $265 \mathrm{~km}$ from Bihar Dar, the capital city of Amhara region. There are one referral hospital, three health centers, and five non-governmental clinics that give different reproductive health services in the town. Only the government referral hospital gives cervical cancer screening services.

\section{Study design}

A community-based cross-sectional study design was used.

\section{Study participants}

The source population was all women age 30-49 years-old who were residents of Debremarkos town. The study population was women age 30-49 years-old who were residents of Debremarkos town during the study period in the selected kebeles. We excluded women who are not permanent residents of the town and those who were critically ill during the data collection period.

\section{Sample size}

The sample size for prevalence was determined based on a single population proportion formula assumption. The magnitude of knowledge and favorable attitude toward cervical cancer screening were $53.7 \%$ and $65.2 \%$ respectively from the previous study in Ethiopia at Hosanna town [17]. A 5\% margin of error was used.

$$
\text { initial sample size for knowledge }=\left(Z \frac{a}{2}\right)^{2} * \frac{p(1-p)}{w 2}=1.96^{2} * \frac{0.537(1-0.537)}{(0.005)^{2}}=382
$$

With considering design effect 1.5 since it had two stages and the sample size was calculated as $382^{*} 1.5=573$ then the non-response rate was also considered to be $10 \%$ and $573^{*} 0.10=57$. Then the final sample size was $573+57=630$.

The sample size for attitude was also calculated similarly.

$$
\text { initial sample size for attitude }=\left(Z \frac{a}{2}\right)^{2} * \frac{p(1-p)}{w 2}=1.96^{2} * \frac{0.652(1-0.652)}{(0.005)^{2}}=349
$$

Considering design effect 1.5 since it had two stages and the sample size was calculated as $349^{*} 1.5=524$ then the non-response rate was also considered to be $10 \%$ and $524^{*} 0.10=52$. Then the final sample size was $524+52=576$. We took the larger sample size which was 630 .

\section{Sampling techniques}

A multistage sampling technique was used and firstly all the kebeles found in the Debremarkos town were listed in a frame. 
Then three out of the seven kebeles were selected by the lottery method. Again the list of households found and coded in each kebele. The size of households consisting of the eligible population to be selected from each kebele was determined proportionally based on the size of the study units and the $k^{\text {th }}$ value was computed for each selected kebele. The women of the selected household were interviewed and if there was more than one woman in the household, the lottery method has been used to select only one. In the case of absenteeism, after three repeated visits the next eligible woman was included in the study.

\section{Study variables}

Dependent variables: Knowledge and attitude of cervical cancer screening.

Independent variables: Socio-Demographic Characteristics, Reproductive and behavioral characteristics.

\section{Operational definitions}

Knowledgeable: Women who answered knowledge questions score of mean value or above were considered as knowledgeable.
Favorable attitude: Women who answered attitude questions with a score of mean value or above were considered to have a favorable attitude.

Multiple sexual partners: Those women who have ever had penetrative sexual intercourse with more than one partner in their life serially or at the same time [18].

Cigarette smoking: The active smoking or ever had a smoking history of women one or more manufactured or hand-rolled tobacco cigarettes per day which excludes passive smokers [19].

\section{Data Collection and Data Quality Control}

To assure the data quality, data were collected with faceto-face interviews by three trained diploma Midwives after two-day data collection training was given to them together with three BSc holder supervisors. The questionnaire was structured and pre-tested which was first prepared in English and translated to local (Amharic) language and then again translated back to English. A pretest was conducted on 32 women of the sample size in Dembecha town and the necessary correction on the tool was employed accordingly.

Table 1: Sociodemographic characteristics of women $(n=630)$ age 30-49 in Debremarkos town, Northwest Ethiopia, 2020.

\begin{tabular}{|c|c|c|}
\hline Variable & Frequency & Percent \\
\hline \multicolumn{3}{|l|}{ Age of mothers } \\
\hline $30-39$ & 509 & 80.8 \\
\hline $40-49$ & 121 & 19.2 \\
\hline \multicolumn{3}{|l|}{ Marital status } \\
\hline Married & 504 & 80 \\
\hline Others ${ }^{*}$ & 126 & 20 \\
\hline \multicolumn{3}{|l|}{ Religion } \\
\hline Christian & 601 & 95.4 \\
\hline Muslim and protestant & 29 & 4.6 \\
\hline \multicolumn{3}{|l|}{ Educational status } \\
\hline No formal education & 176 & 27.9 \\
\hline Primary education & 149 & 23.7 \\
\hline Secondary education & 168 & 26.7 \\
\hline College and above & 137 & 21.7 \\
\hline \multicolumn{3}{|l|}{ Ethnicity } \\
\hline Amhara & 619 & 98.3 \\
\hline Others $^{* *}$ & 11 & 1.7 \\
\hline \multicolumn{3}{|l|}{ Occupation } \\
\hline Housewife & 119 & 18.9 \\
\hline Self-employee (doing own small business) & 223 & 35.4 \\
\hline Private employee(salaried in the nongovernmental sector) & 142 & 22.5 \\
\hline Government employee & 146 & 23.2 \\
\hline \multicolumn{3}{|l|}{ Household income ${ }^{* * *}$} \\
\hline$<900$ & 131 & 20.8 \\
\hline $900-1600$ & 129 & 20.5 \\
\hline $1601-2699$ & 239 & 37.9 \\
\hline$>=2700$ & 131 & 20.8 \\
\hline
\end{tabular}

"Single, divorced and widowed; ${ }^{* *}$ Oromo and Gurage; ${ }^{* * *}$ In Ethiopian Birr 
Citation: Aynalem BY, Anteneh KT, Enyew MM (2021) Knowledge and Attitudes Regarding Cervical Cancer Screening among Women in Debre Markos Town, Amhara Region, Northwest Ethiopia: Community Based Cross-Sectional Study. J Nurs Pract 4(1):240-248

\section{Data processing and analysis}

Epi Info version 7 software was used for data entry and SPSS version 25 for used for analysis. Bivariate logistic regression was employed to identify an association between independent and dependent variables. Variables having a P-value of less than 0.25 in the bivariate logistic regression analysis were fitted into the multivariable logistic regression model. The 95\% confidence interval of Adjusted Odds Ratio (AOR) was computed and a variable having P-value less than 0.05 in the multivariable logistic regression analysis was considered as statistically significant.

\section{Results}

\section{Socio-demographic characteristics of the re- spondents}

Table 2: Reproductive characteristics of women $(n=630)$ age 30-49 in Debremarkos town, Northwest Ethiopia, 2020.

\begin{tabular}{|c|c|c|}
\hline Variable & Frequency & Percent \\
\hline $\begin{array}{l}\text { Age started sexual intercourse (in years) } \\
\begin{array}{l}<=16 \\
>16\end{array}\end{array}$ & $\begin{array}{c}138 \\
492\end{array}$ & $\begin{array}{l}21.9 \\
78.1\end{array}$ \\
\hline $\begin{array}{l}\text { Multiple sexual partners } \\
\qquad \begin{array}{l}\text { No } \\
\text { Yes }\end{array}\end{array}$ & $\begin{array}{l}376 \\
254\end{array}$ & $\begin{array}{l}59.7 \\
40.3\end{array}$ \\
\hline $\begin{array}{l}\text { History of smoking } \\
\text { No } \\
\text { Yes }\end{array}$ & $\begin{array}{l}617 \\
13\end{array}$ & $\begin{array}{l}97.9 \\
2.1\end{array}$ \\
\hline $\begin{array}{l}\text { History of STD } \\
\text { No } \\
\text { Yes }\end{array}$ & $\begin{array}{l}542 \\
88\end{array}$ & $\begin{array}{l}86 \\
14\end{array}$ \\
\hline $\begin{array}{l}\text { Ever use a modern FP method } \\
\qquad \begin{array}{l}\text { No } \\
\text { Yes }\end{array}\end{array}$ & $\begin{array}{l}102 \\
528\end{array}$ & $\begin{array}{l}16.2 \\
83.8\end{array}$ \\
\hline $\begin{array}{l}\text { Duration of modern FP method use } \\
\begin{array}{l}1-3 \text { years } \\
>=4 \text { years }\end{array}\end{array}$ & $\begin{array}{l}425 \\
103\end{array}$ & $\begin{array}{l}80.6 \\
19.4\end{array}$ \\
\hline $\begin{array}{l}\text { Family history of cervical cancer } \\
\text { No } \\
\text { Yes }\end{array}$ & $\begin{array}{l}560 \\
70\end{array}$ & $\begin{array}{l}88.9 \\
11.1\end{array}$ \\
\hline $\begin{array}{l}\text { Ever had got pregnant } \\
\qquad \begin{array}{l}\text { No } \\
\text { Yes }\end{array}\end{array}$ & $\begin{array}{l}105 \\
525\end{array}$ & $\begin{array}{l}16.7 \\
83.3\end{array}$ \\
\hline $\begin{array}{r}\text { Gravidity } \\
\begin{array}{r}1-5 \\
>5\end{array}\end{array}$ & $\begin{array}{l}408 \\
117\end{array}$ & $\begin{array}{l}77.3 \\
22.7\end{array}$ \\
\hline $\begin{array}{l}\text { Ever had given birth } \\
\qquad \begin{array}{l}\text { No } \\
\text { Yes }\end{array}\end{array}$ & $\begin{array}{l}123 \\
507\end{array}$ & $\begin{array}{l}19.6 \\
80.4\end{array}$ \\
\hline $\begin{array}{l}\text { Parity } \\
\begin{array}{r}1-5 \\
>5\end{array}\end{array}$ & $\begin{array}{l}418 \\
89\end{array}$ & $\begin{array}{l}82.4 \\
17.6\end{array}$ \\
\hline
\end{tabular}

All 630 study participants responded to the questionnaire, giving a response rate of $100 \%$. The majorities of women were Amhara 619 (98.3\%), Christian religion 601 (95.4\%), and married 504 (80\%). Four hundred fifty-four $(72.1 \%)$ of the women have attended formal education (Table 1 ).

\section{Reproductive and behavioral characteristics}

One hundred thirty-eight (21.9\%) women had first sexual intercourse at age 16 and below. Two hundred fifty-four (40.3\%) women had a history of multiple sexual partners (MSPs). And 88 (14\%) had a history of the sexually transmitted disease (STD). Around seventy (11.1\%) of respondents had a family history of cervical cancer and $13(2.1 \%)$ of the respondents had a smoking history (Table 2 ).

Table 3: Knowledge about cervical cancer screening among women $(n=630)$ in Debremarkos town, Northwest Ethiopia, 2020.

\begin{tabular}{|c|c|c|}
\hline Variable & Frequency & Percent \\
\hline $\begin{array}{l}\text { Ever heard about cervical cancer } \\
\qquad \begin{array}{l}\text { No } \\
\text { Yes }\end{array}\end{array}$ & $\begin{array}{l}221 \\
409\end{array}$ & $\begin{array}{l}35.1 \\
64.9\end{array}$ \\
\hline $\begin{array}{l}\text { Ever heard about cervical cancer } \\
\text { screening } \\
\text { No } \\
\text { Yes }\end{array}$ & $\begin{array}{l}221 \\
409\end{array}$ & $\begin{array}{l}35.1 \\
64.9\end{array}$ \\
\hline $\begin{array}{l}\text { Knew health institutions that give cervical } \\
\text { cancer screening service } \\
\text { No } \\
\text { Yes }\end{array}$ & $\begin{array}{l}281 \\
349\end{array}$ & $\begin{array}{l}44.6 \\
55.4\end{array}$ \\
\hline $\begin{array}{l}\text { Knew symptoms of cervical cancer } \\
\text { No } \\
\text { Yes }\end{array}$ & $\begin{array}{l}565 \\
65\end{array}$ & $\begin{array}{l}89.3 \\
10.3\end{array}$ \\
\hline $\begin{array}{l}\text { Bleeding during sexual intercourse may } \\
\text { be one of the signs of cervical cancer } \\
\text { No } \\
\text { Yes }\end{array}$ & $\begin{array}{l}569 \\
61\end{array}$ & $\begin{array}{l}90.3 \\
9.7\end{array}$ \\
\hline $\begin{array}{l}\text { Cervical cancer is a killer disease } \\
\text { No } \\
\text { Yes }\end{array}$ & $\begin{array}{c}199 \\
431\end{array}$ & $\begin{array}{l}31.6 \\
68.4\end{array}$ \\
\hline $\begin{array}{l}\text { Is cervical cancer a preventable disease } \\
\text { No } \\
\text { Yes }\end{array}$ & $\begin{array}{l}266 \\
364\end{array}$ & $\begin{array}{l}42.2 \\
57.8\end{array}$ \\
\hline $\begin{array}{l}\text { Is cervical cancer a curable disease } \\
\text { No } \\
\text { Yes }\end{array}$ & $\begin{array}{l}257 \\
373\end{array}$ & $\begin{array}{l}40.8 \\
59.2\end{array}$ \\
\hline $\begin{array}{l}\text { May have cervical cancer without any sign } \\
\text { and symptom } \\
\text { No } \\
\text { Yes }\end{array}$ & $\begin{array}{l}342 \\
288\end{array}$ & $\begin{array}{l}54.3 \\
45.7\end{array}$ \\
\hline Overall knowledge & Frequency & Percent \\
\hline $\begin{array}{l}\text { Knowledgeable } \\
\text { Not Knowledgeable }\end{array}$ & $\begin{array}{l}374 \\
256\end{array}$ & $\begin{array}{l}59.4 \\
40.6\end{array}$ \\
\hline
\end{tabular}


Citation: Aynalem BY, Anteneh KT, Enyew MM (2021) Knowledge and Attitudes Regarding Cervical Cancer Screening among Women in Debre Markos Town, Amhara Region, Northwest Ethiopia: Community Based Cross-Sectional Study. J Nurs Pract 4(1):240-248

\section{Knowledge and attitude towards cervical cancer screening}

About 374 (59.4\%) of respondents were knowledgeable about cervical cancer screening [95\% Cl: 55.5, 63] (Table 3).

There hundred eighty-five (61.1\%) had a favorable attitude towards cervical cancer screening [95\% Cl: 57.4, 65] (Table 4).

\section{Factors associated with knowledge of cervical cancer screening}

After controlling the effect of other variables with binary logistic regression, age, marital status, ethnicity, educational status, income, occupation, history of MSP, history of STD, modern FP method use, and smoking history were significantly associated with knowledge of cervical cancer screening (P-values < 0.25).

After controlling the effect of other variables with mul- tivariable logistic regression analysis history of MSP [AOR: 1.768 (95\% Cl: 1.227, 2.549)], and modern FP method use [AOR: 2.238 (95\% Cl: 1.410, 3.554)] were significantly associated with knowledge of cervical cancer screening (Table 5).

\section{Factors associated with the attitude towards cer- vical cancer screening}

Similarly, with controlling the effect of other variables in binary logistic regression, income, marital status, educational status, age at first sex, modern FP method use, duration of modern FP use, gravidity, and history of STDs were significantly associated with the attitude towards cervical cancer screening ( $P$-values $<0.25)$.

And with controlling the effect of other variables in multivariable logistic regression analysis, an education level (College and above education level [AOR: 2.006, 95\% Cl: 1.147, 3.508)]) marital status [AOR: $2.101(95 \% \mathrm{Cl}: 1.219,3.620)]$, and gravidity [AOR: $1.830(95 \% \mathrm{Cl}: 1.125,2.976)]$, were sig-

Table 4: Attitude towards cervical cancer and its screening among women $(n=630)$ in Debremarkos town, Northwest Ethiopia, 2020.

\begin{tabular}{|c|c|c|c|c|c|c|}
\hline \multirow[t]{3}{*}{ Variables } & \multicolumn{6}{|c|}{ Level of agreement } \\
\hline & \multicolumn{2}{|l|}{ Agree } & \multicolumn{2}{|l|}{ Disagree } & \multicolumn{2}{|c|}{ Indifferent } \\
\hline & Number & Percent & Number & Percent & Number & Percent \\
\hline $\begin{array}{l}\text { Any reproductive age woman is susceptible to } \\
\text { develop cervical cancer }\end{array}$ & 320 & 47.5 & 148 & 23.5 & 162 & 25.7 \\
\hline $\begin{array}{l}\text { Like any women, you are susceptible to develop } \\
\text { cervical cancer }\end{array}$ & 299 & 47.5 & 170 & 27 & 161 & 25.6 \\
\hline Cervical Cancer can be transmitted genetically & 93 & 14.8 & 354 & 56.2 & 183 & 29 \\
\hline Cervical cancer may be dangerous & 383 & 60.8 & 133 & 21.1 & 114 & 18.1 \\
\hline $\begin{array}{l}\text { Precancerous cervical screening may be beneficial } \\
\text { to health }\end{array}$ & 387 & 61.4 & 112 & 17.8 & 131 & 20.8 \\
\hline Cervical cancer screening is painful & 359 & 57 & 109 & 17.3 & 162 & 25.7 \\
\hline Overall attitude & \multicolumn{3}{|c|}{ Frequency } & \multicolumn{3}{|l|}{ percent } \\
\hline Favorable attitude & \multicolumn{3}{|l|}{385} & \multicolumn{3}{|l|}{61.1} \\
\hline Unfavorable attitude & \multicolumn{3}{|l|}{245} & \multicolumn{3}{|l|}{38.9} \\
\hline
\end{tabular}

Table 5: Bivariable and Multivariable analysis of factors associated with knowledge about cervical cancer screening among women in Debremarkos town, Northwest Ethiopia, 2020.

\begin{tabular}{|c|c|c|c|c|}
\hline \multirow[t]{2}{*}{ Variable } & \multicolumn{2}{|l|}{ Knowledge } & \multirow[t]{2}{*}{ Crude OR [95\%Cl] } & \multirow[t]{2}{*}{ AOR [95\%Cl] } \\
\hline & Knowledgeable & Not Knowledgeable & & \\
\hline \multicolumn{5}{|l|}{ Age of mothers } \\
\hline $40-49$ & 65 & 56 & $1.331(0.893,1.984)$ & \\
\hline $30-39$ & 309 & 200 & 1 & \\
\hline \multicolumn{5}{|l|}{ Marital status } \\
\hline Others $^{*}$ & 64 & 62 & 1. $55(1.05,2.29)$ & $0.746(0.487,1.141)$ \\
\hline Married & 310 & 194 & 1 & 1 \\
\hline \multicolumn{5}{|l|}{ Religion } \\
\hline Muslim & 19 & 10 & $0.76(0.35,1.66)$ & \\
\hline Christian and protestant & 355 & 246 & 1 & \\
\hline
\end{tabular}


Citation: Aynalem BY, Anteneh KT, Enyew MM (2021) Knowledge and Attitudes Regarding Cervical Cancer Screening among Women in Debre Markos Town, Amhara Region, Northwest Ethiopia: Community Based Cross-Sectional Study. J Nurs Pract 4(1):240-248

\begin{tabular}{|c|c|c|c|c|}
\hline \multicolumn{5}{|l|}{ Educational status } \\
\hline Primary education & 103 & 46 & $0.68(0.427,1.07)$ & \\
\hline Secondary education & 91 & 77 & $1.28(0.84,1.97)$ & \\
\hline College and above & 74 & 66 & $1.35(0.821,2.03)$ & \\
\hline No formal education & 106 & 70 & 1 & \\
\hline \multicolumn{5}{|l|}{ Ethnicity } \\
\hline Others $^{* *}$ & 9 & 2 & $0.319(0.068,1.49)$ & \\
\hline Amhara & 365 & 254 & 1 & \\
\hline \multicolumn{5}{|l|}{ Occupation } \\
\hline Self-employee & 122 & 101 & $1.36(0.864,2.15)$ & \\
\hline Private employee & 98 & 44 & $0.74(0.442,1.234)$ & \\
\hline Government employee & 80 & 66 & $1.357(0.828,3.430)$ & \\
\hline Housewife & 74 & 45 & 1 & \\
\hline \multicolumn{5}{|l|}{ Household income ${ }^{* * *}$} \\
\hline $900-1600$ & 62 & 67 & $1.24(0.762,2.018)$ & \\
\hline $1601-2699$ & 144 & 95 & $0.757(0.492,1.164)$ & \\
\hline$>=2700$ & 98 & 33 & $0.386(0.245,2.22)$ & \\
\hline$<900$ & 70 & 61 & 1 & \\
\hline $\begin{array}{l}\text { Age started sexual interco } \\
\text { time } \\
\quad<=16 \\
\quad>16\end{array}$ & $\begin{array}{l}77 \\
297\end{array}$ & $\begin{array}{l}61 \\
195\end{array}$ & $\begin{array}{l}1.207(0.824,1.767) \\
1\end{array}$ & \\
\hline $\begin{array}{l}\text { Multiple sexual partners } \\
\text { Yes } \\
\text { No }\end{array}$ & $\begin{array}{l}178 \\
196\end{array}$ & $\begin{array}{l}76 \\
180\end{array}$ & $\begin{array}{l}2.15(1.54,3.011) \\
1\end{array}$ & $\begin{array}{l}1.768(1.23,2.55) \\
1\end{array}$ \\
\hline $\begin{array}{l}\text { History of smoking } \\
\qquad \begin{array}{l}\text { Yes } \\
\text { No }\end{array}\end{array}$ & $\begin{array}{l}9 \\
247\end{array}$ & $\begin{array}{l}4 \\
370\end{array}$ & $\begin{array}{l}3.37(1.03,11.07) \\
1\end{array}$ & $\begin{array}{l}3.057(0.896,10.429) \\
1\end{array}$ \\
\hline $\begin{array}{l}\text { History of STD } \\
\begin{array}{l}\text { Yes } \\
\text { No }\end{array}\end{array}$ & $\begin{array}{l}48 \\
208\end{array}$ & $\begin{array}{l}40 \\
334\end{array}$ & $\begin{array}{l}1.926(1.22,3.034) \\
1\end{array}$ & $\begin{array}{l}1.446(0.875,2.389) \\
1\end{array}$ \\
\hline $\begin{array}{l}\text { Ever use a modern FP metl } \\
\text { Yes } \\
\text { No }\end{array}$ & $\begin{array}{l}328 \\
46\end{array}$ & $\begin{array}{l}200 \\
56\end{array}$ & $\begin{array}{l}1.997(1.30,3.06) \\
1\end{array}$ & $\begin{array}{l}2.238(1.41,3.55) \\
1\end{array}$ \\
\hline $\begin{array}{l}\text { Duration of modern FP me } \\
\qquad \begin{array}{c}> \\
\text { years }\end{array} \\
1-3 \text { years }\end{array}$ & $\begin{array}{l}65 \\
263\end{array}$ & $\begin{array}{l}37 \\
162\end{array}$ & $\begin{array}{l}1.08(0.691,1.695) \\
1\end{array}$ & \\
\hline $\begin{array}{l}\text { Family history of cervical c } \\
\qquad \begin{array}{l}\text { Yes } \\
\text { No }\end{array}\end{array}$ & $\begin{array}{l}45 \\
329\end{array}$ & 25 & $\begin{array}{l}1.26(1.54,2.12) \\
1\end{array}$ & \\
\hline $\begin{array}{l}\text { Ever had got pregnant } \\
\text { Yes } \\
\text { No }\end{array}$ & $\begin{array}{l}319 \\
55\end{array}$ & $\begin{array}{l}206 \\
50\end{array}$ & $\begin{array}{l}1.41(0.924,2.145) \\
1\end{array}$ & \\
\hline
\end{tabular}


Citation: Aynalem BY, Anteneh KT, Enyew MM (2021) Knowledge and Attitudes Regarding Cervical Cancer Screening among Women in Debre Markos Town, Amhara Region, Northwest Ethiopia: Community Based Cross-Sectional Study. J Nurs Pract 4(1):240-248

\begin{tabular}{|c|l|l|l|}
\hline $\begin{array}{c}\text { Gravidity } \\
>5\end{array}$ & 247 & 161 & $0.959(0.629,1.46)$ \\
$1-5$ & 72 & 45 & 1 \\
\hline $\begin{array}{c}\text { Ever had given birth } \\
\text { Yes }\end{array}$ & 309 & 197 & $1.42(0.959,2.114)$ \\
No & 65 & 59 & 1 \\
\hline $\begin{array}{c}\text { Parity } \\
>5\end{array}$ & 56 & 33 & $1.096(0.683,1.758)$ \\
\hline $1-5$ & 254 & 164 & 1 \\
\hline
\end{tabular}

"single, divorced and widowed; ${ }^{* *}$ Oromo and Gurage; ${ }^{* * *}$ In Ethiopian Birr

Table 6: Bivariable and Multivariable analysis of factors associated with an attitude towards cervical cancer screening among women in Debremarkos town, Northwest Ethiopia, 2019.

\begin{tabular}{|c|c|c|c|c|}
\hline \multirow[t]{2}{*}{ Variable } & \multicolumn{2}{|l|}{ Attitude } & \multirow[t]{2}{*}{ Crude OR $[95 \% \mathrm{Cl}]$} & \multirow[t]{2}{*}{ AOR $[95 \% \mathrm{Cl}]$} \\
\hline & Favorable & Un Favorable & & \\
\hline \multicolumn{5}{|l|}{ Marital status } \\
\hline Married & 323 & 181 & 1 & 1 \\
\hline Others ${ }^{*}$ & 62 & 64 & $1.842(1.242,2.73)$ & $2.101(1.22,3.62)$ \\
\hline \multicolumn{5}{|l|}{ Educational status } \\
\hline Primary education & 99 & 50 & $0.862(0.546,1.363)$ & \\
\hline Secondary education & 100 & 68 & $1.161(0.752,1.793)$ & \\
\hline College and above & 75 & 52 & $1.412(0.896,2.225)$ & $2.01(1.15,3.51)$ \\
\hline No formal education & 111 & 65 & 1 & 1 \\
\hline \multicolumn{5}{|l|}{ Household income ${ }^{* * *}$} \\
\hline $900-1600$ & 72 & 57 & $1.62(0.979,2.68)$ & \\
\hline $1601-2699$ & 141 & 89 & $1.422(0.910,2.223)$ & \\
\hline$>=2700$ & 84 & 47 & $1.145(0.687,1.908)$ & \\
\hline$<900$ & 88 & 43 & 1 & \\
\hline \multicolumn{5}{|l|}{ Age at first sex } \\
\hline$<=16$ & 76 & 62 & $1.377(0.940,2.018)$ & \\
\hline$>16$ & 309 & 183 & 1 & \\
\hline \multicolumn{5}{|l|}{ History of STD } \\
\hline Yes & 44 & 44 & $1.697(1.08,2.668)$ & $1.28(0.719,2.282)$ \\
\hline No & 201 & 341 & 1 & 1 \\
\hline \multicolumn{5}{|c|}{ Ever use a modern FP method } \\
\hline Yes & 336 & 192 & $1.893(1.235,2.90)$ & $1.244(0.709,2.18)$ \\
\hline No & 49 & 53 & 1 & 1 \\
\hline \multicolumn{5}{|c|}{ Duration of modern FP method usage } \\
\hline$>3$ years & 58 & 44 & $0.704(0.454,1.093)$ & \\
\hline $1-3$ years & 277 & 148 & 1 & \\
\hline \multicolumn{5}{|l|}{ Gravidity } \\
\hline$>5$ & 54 & 63 & $1.659(1.09,0.517)$ & $1.830(1.125,2.98)$ \\
\hline $1-5$ & 139 & 269 & 1 & 1 \\
\hline
\end{tabular}

"single, widowed, divorced; ${ }^{* * *}$ In Ethiopian Birr

nificantly associated with the attitude towards cervical cancer screening (Table 6).

\section{Discussion}

This study was done to investigate knowledge and at- titude towards cervical cancer screening among women in Debremarkos town, northwest Ethiopia. So, the study found that $59.4 \%$ [95\% Cl: $55.5,63]$ and $61.1 \%$ [95\% Cl: $57.4,65]$ of respondents were knowledgeable and had a favorable attitude towards CC screening respectively. 
Citation: Aynalem BY, Anteneh KT, Enyew MM (2021) Knowledge and Attitudes Regarding Cervical Cancer Screening among Women in Debre Markos Town, Amhara Region, Northwest Ethiopia: Community Based Cross-Sectional Study. J Nurs Pract 4(1):240-248

The finding of this research was lower than the studies done in Ethiopia (91\%) [20]. The possible explanation for this might be the difference in the level of knowledge as evidenced by a study done in Ethiopia on health care providers.

The finding of this study was also higher than studies done in China (19.5\%) [21], Kuwait (52.3\%) [22], at Finote-Selam, Ethiopia (30.3\%) [23], in Addis Ababa, Ethiopia (23.4\%) [24], at Hadiya Zone (53.7\%) [17], and Southern Ethiopia (77.1\%) [25], The possible explanation might be; the difference in the study area and study population as evidenced by a study done in Ethiopia on the both urban and rural population that was different from our study population and study area which was urban, and unavailability of screening center near the community as evidenced by a study done a Finote-Selam town.

As shown in this study, a woman who had a history of MSPs was an important factor for knowledge on cervical cancer screening. Women who have had a history of MSPs were 1.768 times more likely knowledgeable on cervical cancer screening as compared with those who did not MSP [AOR: $1.768(95 \% \mathrm{Cl}: 1.227,2.549)]$. This study was supported by the result of a study done in China [21] and Kuwait [22]. The possible explanation might be women, who had MSP history, would have the chance to be infected with STIs with its signs and symptoms which increases health facility visits and availability of the screening service.

The current study result showed that a woman who had used the modern family planning method was another important factor for knowledge of cervical cancer screening. Women who have ever used the modern family planning method were 2.238 times more likely knowledgeable on cervical cancer screening as compared to those who did not use [AOR: 2.238 (95\% Cl: 1.410, 3.554)]. This result was supported by the finding from Malaysia [26] and China [21]. The above association might be explained by women who have used the modern family planning method, may have a chance of getting an education on CC screening.

The study finding of attitude towards cervical cancer screening (61.1\%) was in-line with similar reports at Finote-Selam, Ethiopia (58.1\%) [23]. The finding of this research was lower than the studies done in Zimbabwe (71.2\%) [27]. The possible explanation might be the difference in the study area and study population which was done nationwide in religious areas.

The finding of this study was higher than studies done at Hadiya Zone, and South Ethiopia (53.7\%) [17] and in Kuwait (30.6\%) [22]. The possible explanation might be the difference in the study area and study population as evidenced by a study done in Ethiopia on the both urban and rural population that was different from our study population and study area which was urban; and unavailability of screening service near the community as evidenced b study done a Finote-Selam town.

As shown in this study, marital status was a significant predictor of attitude towards cervical cancer screening. This study showed that women who were single/divorced/widowed 2.101 times more likely had a favorable attitude to- wards cervical cancer screening as compared with married women [AOR: 2.101 (95\% Cl: 1.219, 3.620)]. No study supported this finding. The possible explanation for this might be single, divorced, or widowed women may have MSP which causes sexually transmitted disease. His may lead the women to have more visit health facilities which increases the chance to have a good attitude towards CC screening.

Educational status was also the main significant factor for attitude towards CC screening. Women who took college \& above education were 2.01 times more likely to have a favorable attitude towards CC screening as compared with women who did not take formal education [AOR: $2.01,95 \% \mathrm{Cl}$ : $1.147,3.508)]$. This study was supported by the study done at Finote-Selam town in Ethiopia [23] and in Kuwait [22]. This might be explained with, as the level of education increases, the women will have the chance to know about cervical cancer screening which can change the attitude towards CC screening. The current study result also showed that women who had more than five pregnancies were 1.830 times more likely to have a favorable attitude towards CC screening as compared to those who had less than five pregnancies [AOR: 1.830 (95\% Cl: $1.125,2.976)]$. No study supported this finding. The above association might be explained by women who have had a large number of pregnancies, may have many health institution visits for antenatal care service or pregnancy-related complications. This may increase the chance of getting information on $\mathrm{CC}$ screening which may change their attitude.

\section{Conclusion}

This study showed the magnitude of knowledge and attitude towards cervical cancer screening was low. Modern family planning method use and history of multiple sexual partners were significant factors for knowledge of cervical cancer screening. Similarly, education level, marital status, and gravidity were significant factors for attitude towards cervical cancer screening.

\section{Declarations}

\section{Availability of data and materials}

Data supporting this study can be obtained on request.

\section{Ethical clearance}

Ethical clearance was obtained from the Ethical Review Committee of the Department of Midwifery, under the delegation of the institutional review board of the University of Gondar. Ethical clearance and formal letters were also obtained from the University Gondar School of Midwifery and were submitted to Debremarkos health office and permissions were obtained. Finally, written informed consent was also obtained from each study participant.

\section{Acknowledgments}

We would like to thank the University of Gondar and Debremarkos health office for their permission to this research and we gratefully acknowledge all study individuals for their participation in the study. 
Citation: Aynalem BY, Anteneh KT, Enyew MM (2021) Knowledge and Attitudes Regarding Cervical Cancer Screening among Women in Debre Markos Town, Amhara Region, Northwest Ethiopia: Community Based Cross-Sectional Study. J Nurs Pract 4(1):240-248

\section{Funding}

The study was funded by the University of Gondar. The university supported the study by ensuring ethical acceptability and financially covered the costs for data collectors' training and data collectors' per diem costs during data collection.

\section{Consent for publication}

All the authors have a copy of this manuscript. The authors would like to confirm that this article has not been submitted to any other journal and we fully agree to be published by this journal.

\section{Authors' contributions}

BY: proposed the initial idea for the study; BY, KT, MM: Contributed to the study design; BY: Collected all the data; all authors analyzed and interpreted the data; BY: Drafted the manuscript; BY, KT and MM: Contributed to the writing of the manuscript. BY, KT, and MM: Prepared the manuscript for publication; all authors read and approved the final manuscript.

\section{Disclosure}

The authors report no conflicts of interest in this work.

\section{References}

1. WHO (2013) Comprehensive cervical cancer prevention and control: A healthier future for girls and women.

2. Health WHOR, Diseases WHOC, Promotion H (2006) Comprehensive cervical cancer control: A guide to essential practice.

3. Agency BC (2011) Cervical cancer screening program, annual report.

4. ACCP (2004) Planning and implementing cervical cancer prevention and control programs: A manual for managers. WHO.

5. UNIATF (2016) UN Joint Global Programme on Cervical Cancer Prevention and Control. In: The United Nations Global Cervical Cancer Programme October, 2016.

6. Simon AE, Waller J, Robb K, Wardle J (2010) Patient delay in presentation of possible cancer symptoms: The Contribution of knowledge and attitudes in a population sample from the United Kingdom. Cancer Epidemiol Biomarkers Prev 19: 2272-2277.

7. WHO (2012) Cryosurgical equipment for the treatment of precancerous cervical lesions and prevention of cervical cancer.

8. Moyer VA (2012) Screening for cervical cancer: US preventive services task force recommendation statement. Annals of internal medicine 156: 880-891.

9. Black E, Richmond R (2018) Prevention of cervical cancer in Sub-Saharan Africa: The advantages and challenges of HPV vaccination. Vaccines (Basel) 6: 61.

10. Bruni L B-RL, Albero G, Serrano B, et al. (2019) Human Papillomavirus and Related Diseases in Ethiopia.

11. Sawaya GF (2009) Cervical-cancer screening-new guidelines and the balance between benefits and harms. New England Journal of Medicine 361: 2503-2505.

12. De Sanjosé S, Serrano B, Castellsagué X, et al. (2012) Human papillomavirus (HPV) and related cancers in the Global Alliance for Vaccines and Immunization (GAVI) countries. A WHO/ICO HPV information centre report. Vaccine 30: 1-83.
13. Rosser JI, Njoroge B, Huchko MJ (2015) Knowledge about cervical cancer screening and perception of risk among women attending outpatient clinics in rural Kenya. Int J Gynaecol Obstet 128: 211-215.

14. Ali-Risasi C, Mulumba P, Verdonck K, et al. (2014) Knowledge, attitude and practice about cancer of the uterine cervix among women living in Kinshasa, the Democratic Republic of Congo. BMC Womens Health 14: 30.

15. WHO (2013) Guidelines for screening and treatment of precancerous lesions for cervical cancer prevention: supplemental material.

16. Bayu H, Berhe Y, Mulat A, et al. (2015) Cervical cancer screening service uptake and associated factors among age eligible women in mekelle zone, northern Ethiopia, 2015: A community based study using health belief model. PLoS One 11: e0149908.

17. Aweke YH, Ayanto SY, Ersado TL (2017) Knowledge, attitude and practice for cervical cancer prevention and control among women of childbearing age in Hossana town, Hadiya zone, Southern Ethiopia: Community-based cross-sectional study. PLoS One 12: e0181415.

18. Kileo NM, Michael D, Neke NM, et al. (2015) Utilization of cervical cancer screening services and its associated factors among primary school teachers in Ilala Municipality, Dar es Salaam, Tanzania. BMC Health Services Research 15: 552.

19. Gelibo T, Roets L, Getachew T, et al. (2017) Coverage and factors associated with cervical cancer screening: Results from a population-based WHO steps study in Ethiopia. Journal of Oncology Research and Treatments 2: 115.

20. Kress CM, Sharling L, Owen-Smith AA, et al. (2015) Knowledge, attitudes, and practices regarding cervical cancer and screening among Ethiopian health care workers. Int J Women's Health 7: 765-772.

21. Di J, Rutherford S, Wu J, et al. (2015) Knowledge of cervical cancer screening among women across different socio-economic regions of China. PLoS One 10: e0144819.

22. Al Sairafi M, Mohamed FA (2009) Knowledge, attitudes, and practice related to cervical cancer screening among Kuwaiti women. Med Princ Pract 18: 35-42.

23. Geremew AB, Gelagay AA, Azale T (2018) Comprehensive knowledge on cervical cancer, attitude towards its screening and associated factors among women aged 30-49 years in Finote Selam town, northwest Ethiopia. Reprod Health 15: 29.

24. Shiferaw S, Addissie A, Gizaw M, et al. (2018) Knowledge about cervical cancer and barriers toward cervical cancer screening among HIV-positive women attending public health centers in Addis Ababa city, Ethiopia. Cancer Medicine 7: 903-912.

25. Dulla D, Daka D, Wakgari N (2017) Knowledge about cervical cancer screening and its practice among female health care workers in southern Ethiopia: A cross-sectional study. Int J Women's Health 9: 365-372.

26. Al-Naggar RA, WY Low, Md Isa Z (2010) Knowledge and barriers towards cervical cancer screening among young women in Malaysia. Asian Pacific J Cancer Prev 11: 867-873.

27. Mutambara J, Mutandwa P, Mahapa M, et al. (2017) Knowledge, attitudes and practices of cervical cancer screening among women who attend traditional churches in Zimbabwe. Journal of Cancer Research and Practice 4: 53-58. 